\title{
DATABASE MANAGEMENT FOR INTEGRATED AVIONICS SYSTEM
}

\author{
Ira Glickstein, Steve Ruberg, and Lt John Marsh
}

IBM Federal Systems Division, Owego NY

Wright Laboratory, Wright-Patterson AFB, Ohio

\begin{abstract}
The current push to correlate, associate, and combine data and information from multiple sources will take present avionics architectures to their limits. Real-time database management is one issue that must be resolved to effectively utilize the attributes of the future avionics platform. Future systems will require a distributed database management system (DBMS) that is interfaced with the entire avionics platform.
\end{abstract}

The avionics DBMS will require that data content be maintained independently of the application software. The DBMS must allow the modification of data structures without change to application software. In addition, advanced hardware and software techniques must be applied to improve the performance of DBMS procedures. Utilizing AI knowledge-bases and digital map-based spatio-temporal databases as primary examples, this paper describes the requirements for an avionics DBMS and offers potential design solutions that could be implemented in an integrated avionics architecture.

\section{INTRODUCTION}

Advanced missions, such as precision strike and air superiority and defense, require data from multiple sources to be associated using new inferential and correlation techniques. Timely access to all system data is beyond the limits of current avionics architectures. Therefore, avionics database management is a critical issue that must be resolved to effectively utilize the attributes of future avionics platforms and upgrades to current platforms.

Based on the Functionally Integrated Resource Manager (FIRM) project at Wright Laboratory, and IR\&D projects at IBM, this paper reviews current avionics databases and projects an explosion of future data and knowledgebases. There is a critical need for a real-time, distributed DBMS that is interfaced with the entire avionics platform. Data content must be maintained independently of the application software so that data structures and/or application programs may be modified without requiring a redesign of other parts of the system. In addition, advanced hardware and software techniques must be applied to improve the performance of DBMS procedures. Utilizing $\mathrm{AI}$ knowledgebases and digital map-based spatio-temporal databases as primary examples, we describe the requirements for an avionics DBMS and offer potential design solutions that could be implemented in an integrated avionics architecture.

\section{AVIONICS DATABASE ISSUES}

Avionics databases have traditionally been hard coded into an imbedded processing system. As databases have become more abundant and complex the software procedures used to access them have also increased in complexity. These data procedures are directly accessed using a function call by the application programs that need the data. This close coupling of application programs, data access procedures, and data structures aids real-time operation but detracts from software 
maintainability and reusability. Another issue that contributes to the maintainability problem is the lack of standards for data procedures, data structures and data elements.

Increasing Data Demands

Critical to the high tech weaponry that performed so well in the Persian Gulf War are the many software programs and databases that make situation assessment, threat assessment, and mission success possible. Terrain data combined with sensor data resulted in destroying targets with pinpoint accuracy. Some platforms utilized radar signature data for beyond visual range air target recognition. Communications were carried out using crypto and channel frequency databases. Battle plans were overlaid on digitized charts and electronic warfare sensors utilized threat emitter libraries to determine lethality or jamming methods.

Future avionics systems will be even more dependent on large on-board databases. It will be possible to download weather data at the start of the mission. Geographic, gravitational and magnetic variation data will be used for navigation and low-level missions. Pilot aiding systems will require situation responses from large knowledge databases to be accessed fast enough to allow the information to be acted on. Air and ground target identification will require target signature databases for registration with optical, infrared, laser, or radar sensor inputs.

Increasing Software Complexity

Avionics application programs are becoming much more complex in order to get the maximum amount of information out of the data obtained from sensors and on-bcard databases. Multi-sensor integration and fusion techniques will allow more accurate target acquisition and identification, improved navigation, and better threat detection/location. Expert systems promise even better situation awareness. With traditional military data management techniques, each application has its own copy of the data and imbedded management functions. However, this approach to data management will not work in future integrated systems that will depend on data sharing across sensor subsystems.
Absence of Standardization

The absence of database standards could add more to the growing software maintenance problem. For example, of the several planned terrain database systems being offered, all have different methods for accessing the data and differing file structures to perform similar applications. Also, the lack of data element standards can increase modification and integration costs because of the effect on system inter-operability.

\section{DATABASE MANAGEMENT IN INTEGRATED AVIONICS}

Steps to separate the database functions from the application need to be taken as the complexity of both the databases and the applications increases. Generally both the application and the database will benefit from this division. The application will be less complex and will have more independence from physical storage structures. The database will be easier to design, use, and maintain. [Meisenbacher_87].

Aircraft avionics modular processing systems, as shown in Figure 3-1, provide an opportunity to move away from the file based computing environment of current systems. These integrated systems will provide faster processors with large global memory and faster local memory to further improve access speed and data throughput.

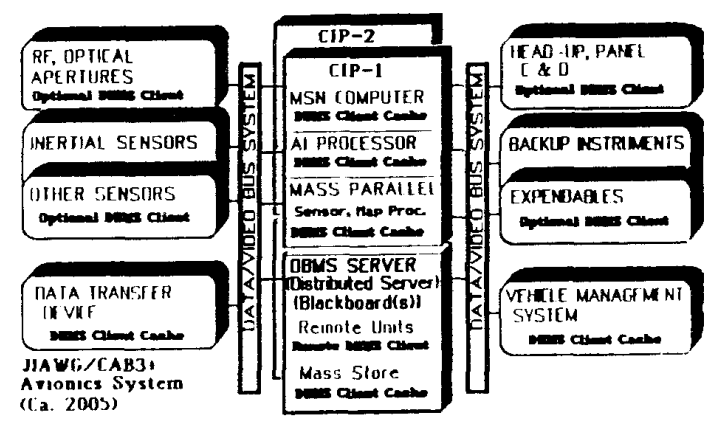

Figure 3-L DBus Chenh/Server Woded Compatible with JaWG Arthitecture 
A large random access global memory will allow data downloaded from the mass memory to be accessed quickly by any processing element. A parallel bus structure will allow fast access to data created by other applications running on separate processing elements. Most importantly, an integrated architecture and its operating system will be essential to exploit the hardware features which will allow the implementation of a capable database management system within an avionics platform.

Processors and Memory

Any effort to provide a DBMS that ensures security, integrity, concurrency, and recovery will impact real-time operation by introducing processing overhead which requires additional processor throughput. The higher processing speeds, distributed or parallel processing, and innovative memory management techniques associated with integrated avionics must be used to overcome this overhead to attain real-time. The DBMS must use its layered interface to the operating system to manage these architectural features in its quest to manage the data. One aspect of this management responsibility would be to ensure that data is in local memory when it is required. This would include juggling the resources of mass storage devices, individual processing element memory, global bulk memory, and a specific processor's own fast local memory with the help of software layers like the operating system. If configured correctly, the DBMS would virtually eliminate the latency penalty of retrieving data directly from a mass storage system, such as an optical disk, by loading potentially useful pages into global bulk memory well in advance of its actual use.

As an example, large areas of terrain data could be stored on-board in an optical disk system. The DBMS would retrieve the current mission area and transfer it to free areas of the global bulk memory, where it would reside until needed for specific processing. Only the data for the current target area would be transferred to the limited local memory resources of a prosessor for very fast or intensive algorithmic work, such as fusion or display processing.

Parallel Bus Structure

A parallel bus structure will provide a processor fast access to all available data. The parallel bus connects all the elements in the integrated architecture, such as global bulk memory, other processor's local memory, and mass storage devices. It is high in data bandwidth and allows the DBMS to perform very fast transactions. This is because there is little overhead between the hardware- based transaction and the DB calls. In other words software layers, such as the operating system, do not add much processing overhead to parallel bus data transfers. As a result, data movement among elements is virtually as fast as the hardware will allow.

Using the terrain example again, the parallel bus ties together the hardware elements and allows efficient data movement. The data travels from the optical disk controller across the parallel bus to the global bulk memory with a DBMS command which utilizes an operating system call. The DBMS then moves necessary smaller target areas over the parallel bus into a processor's local memory where algorithmic work can be done. The results of this can then be transferred over the parallel bus to another processor to be correlated with other-data or displayed.

Integrated Architecture and Operating System Layers

The integrated architecture is the key which links together all vital elements of this system. The elements include processors, memory, the parallel interface bus, and mass storage devices. Although the DBMS does not directly manage these hardware resources, it must efficiently interface to the operating system layer, which does. Although there will be some overhead associated with using the operating system routines, they will serve to buffer the DBMS from the intricate details of hardware operation. Just as the DBMS will handle the details of data management for the application program, the operating system layer handles the hardware management for the DBMS.

In current systems, hardware elements are connected via 1553 databusses, which are serial. The speeds associated with these communications cannot compare to the tightly coupled elements of an integrated architecture, which process and pass data in parallel between hardware elements. This architecture is the power behind the DBMS. Although there is 
overhead associated with a DBMS, this extra software layer will allow faster application code development, reduced software maintenance time, and full utilization of advanced integrated avionics.

\section{DBMS DESIGN SOLUTIONS}

This section discusses top-level design considerations and solution approaches applicable to a range of potential avionics DBMS implementations. The principal requirements and constraints for DBMS design solutions are:

o Compatibility with future highly integrated avionics architectures (JLAWG Common Avionics Baseline ca. 2005).

- Applicable to upgrades to current avionics systems, retaining existing hardware and software to the maximum reasonable extent.

- Common database support for current avionics functions, allowing reuse of existing algorithms and Ada code.

- Support for advanced data association techniques (A I knowledge-base inferencing/pattern-matching and cellular automata spatio-temporal correlation).

o Implementable with advanced software methodology and standard higher order language (object oriented paradigm, Ada).

DBMS Client/Server Model Within JIAWG Architecture

We have selected a distributed, object-oriented, client/server model as the basic approach to a DBMS design solution. As indicated in Figure 3-1, functionally redundant DBMS servers will be located within the central integrated processor (CIP) units of a future JLAWG-compatible avionics architecture. The CIPs must be redundant and physically separated to minimize the possibility of simultaneous battle damage or failure. DBMS client caches, if required, could be located within all local processors and some remote ones. In order to meet real-time performance needs, trade-offs between caching requirements and parallel bus bandwidth capability are being investigated.

Advanced avionics systems, in the time period under consideration, will have at least three different types of processors: 1) Mission computers (JLAWG CAP-16 and CAP-32 performing traditional avionics functions), 2) AI processors (parallel processors performing symbolic pattern-matching and inferencing at rates that make real-time $\mathrm{AI}$ practical), and 3) Array processors (massively parallel multi-dimensional arrays performing sub-symbolic sensor data correlation and situation display generation).

The selected system architecture is a reasonable extension to that being implemented for the Air Force F-22. It reflects the trend for virtually all digital processing to share resources in physically distributed but functionally integrated processors. Sensors, effectors, and display devices will have a minimum of dedicated processors and databases.

Application Programs Utilize DBMS Services Layer

The application program layer, as shown in Figure 4-1, will sit atop a DBMS layer which, for purposes of common data access and management, will insulate the application programs from the operating system (OS) layer and from each other. This is a major departure from today's avionics systems where applications access common data as files in global memory and where a change to any application program is likely to have far-reaching effects on other applications. The layer approach will allow upgrades and additions to be made to application programs, common data, and/or processor hardware without forcing a redesign of the entire system.

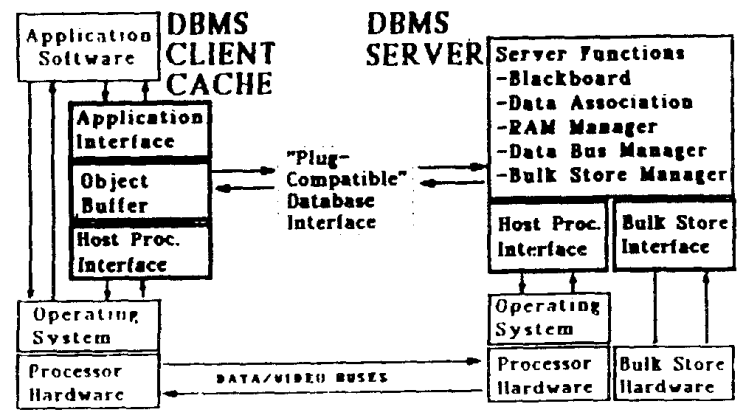

Pigure 4-L Deys Cient Cache Supports RearTime Dath Acoes and "Phue-Computible" Access to Serter

We envision the client cache software as consisting of a standard skeleton, supplied as part of the DBMS, with data schemas and real-time cache protocols for "plug-compatible" 
interface with the DBMS. The client cache skeleton will be customized by hardware suppliers to mate with the particular host processor OS and data/video bus protocols. It will be further customized by application programmers to convert their internal data structures, where necessary, to standard DBMS schemas and data access operations. This will promote software reuse. Applications running in remote units that do not have local client caches will be served via clients located in the server. This will make it economically practical to upgrade existing avionics systems and retain, without modification, older avionics units that still have adequate service life.

A broad selection of data schemas will be provided to support both existing and future applications. We selected the object-oriented paradigm in recognition of the fact that some new applications will take DBMS functionality well beyond the traditional "flat file" construct. However, provision will be made for avionics applications that continue to make use of tables, lists, and other hierarchical and relational data object constructs.

Each common data structure will be encapsulated in an object with a defined interface and access methods. Regardless of the actual structure of the data as stored in a particular implementation or at a particular phase of a mission, a common viewpoint will be provided to the applications that need to access that data.

When an application needs to read or write to a DBMS data object, it need not know if that object is in local cache, in the server, or in compressed form on the bulk storage device. Other than access time, all data transactions will be the same to the application program. To assure real-time access to critical data, the DBMS will support periodic cache update processes that can be set up as a function of mission phase and system parameters. Provisions will be made to assure that concurrency of access to multiple copies of common data will not affect data integrity.

DBMS Blackboard, Data Association and Management Services

The DBMS server will perform blackboard and data association services as well as manage random access memory (RAM), data bus, and bulk store resources.
The blackboard concept is based on the fact that there may be multiple sources for a given piece of information, and multiple applications that need it. The blackboard manager associates data from multiple sources, selects the best current source of the data, and posts it, along with an indication of accuracy, so any application may use it. This insulates application programs from each other and simplifies upgrades. For example, if GPS is added to the system, more accurate present position and other navigation data is automatically available to any application without changing the application program in any way. Should GPS be unavailable, the blackboard manager will automatically select Kalman-filtered Doppler-inertial, or whatever is next best, again without affecting other applications. In addition to Kalman filtering, the blackboard manager will take account of BIT status, Doppler over-water indication, and other quality indicators to determine and post the best present position velocity, and other system parameters of common interest.

Advanced AI processors, currently under development, perform symbolic inferencing in parallel, making real-time AI practical for future avionics systems [Michael 1991, Kogge 1992]. Such processors take data association to the next logical step by instantly comparing thousands of patterns to facts, and demons to patterns. For example, input from a sensor may add to currently known facts, which will trigger a parallel comparison of the left-hand sides of rules and lead to the assertion of new facts. This, in turn, may cause demons that monitor different patterns of facts to trigger appropriate actions by the avionics system to recognize and counter a threat.

Massively parallel processors perform sub-symbolic data correlation in parallel, making real-time multi-dimensional spatio-temporal data correlation practical. The Connection Machine is an example of a class of concurrent processors based on the cellular automata (CA) paradigm [Hillis, 1984]. Such processors are a step beyond the current generation of dedicated systolic array machines utilized for sensor target/threat recognition, and the graphics processors utilized for complex display generation. The ultimate goal is to develop a general-purpose (GP) parallel 
processor that will serve as a shared resource for many different applications.

In addition to signal processing, such GP-parallel processors will perform situation assessment and tactical response functions, using algorithms currently under development at IBM [Glickstein 1991, Stiles 1991]. Although these CA algorithms currently run efficiently on serial and coarse grain parallel processors, they were designed to run on a concurrent machine with thousands of processors, each of which can exchange data with nearest neighbors in a 2-D, 3-D, or higher-dimensional grid. Test runs on an actual Connection Machine indicated orders of magnitude speed-ups. Figure 4-2 indicates how static and dynamic data from multiple sources may be mapped onto a 4-D array such that all data in an $x, y, z, t$ cell is available to an individual processor for correlation. By means of message passing between nearest neighbors, problems of global extent, such as the best route from point $A$ to point $B$, can be determined. We have also used $\mathrm{CA}$ algorithms to compute intervisibility maps and for multi-sensor correlation with digital map terrain and feature data.

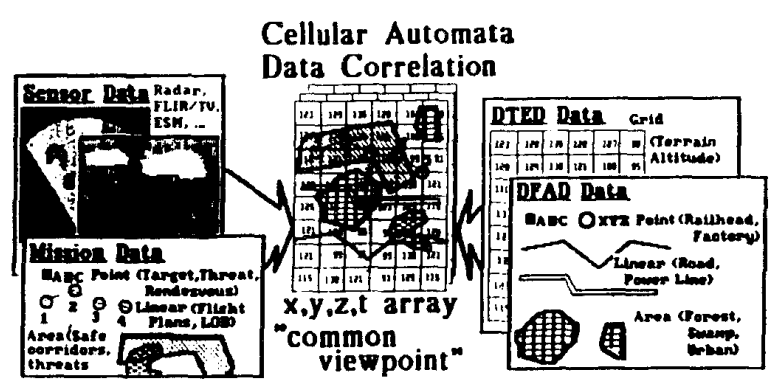

Figure 4-2 Date Correlation besed on CA Paradigm Provida "Common

\section{$\mathrm{V}$ CONCLUSIONS}

We are witnessing an explosion of databases, including AI knowledge bases and sensor/map spatio-temporal databases, that will require a real-time, distributed DBMS that is interfaced with the entire avionics platform. The FIRM project is developing a broad-based, object-oriented DBMS solution that will be applicable to future systems and upgrades to current systems. Given projected advances in on-board sensors and parallel processors, the FIRM DBMS will enable real-time $\mathrm{AI}$ and
CA-based data correlation that will greatly improve our ability to conduct challenging missions, such as precision strike and air superiority and defense. In addition, a standard DBMS will increase software maintainability and reusability, resulting in weapon system lifecycle cost savings.

\section{REFERENCES}

Cattell, R. G. G.; Object Data Management, Addison-Wesley Publishing Company, Inc., 1991.

Glickstein, Ira S.; Stiles, Peter N.; "Situation Assessment Using Cellular Automata Paradigm," 10th Digital Avionics Systems Conference, IEEE/AIAA, Los Angeles, CA, October 1991.

Hillis, W. Daniel; "The Connection Machine: A Computer Architecture Based on Cellular Automata," Physica 10D (1984).

Kogge, Peter M.; Giambra, Timothy W; Mastranadi, John F.; "Real-Time Al System," Report \# C93-92-126, IBM Federal Sector Division, Owego, NY, January 1992.

Meisenbacher, John; "DBMS Technology for Distributed Real-Time Data Intensive Systems," Proceeding of NAECON 1987, IEEE Dayton Section, May 1987.

Michael, Mark; Kogge, Peter M.; Dennis, Charles A.; Utt, James; "Parallel Processing Tutorial," NAECON '91, IEEE Dayton Section, 20 May 1991.

Stiles, Peter N.; Glickstein, Ira S.; "Route Planning," 10th Digital Avionics Systems Conference, IEEE/AIAA, Los Angeles, CA, October 1991. 